\title{
Correlação entre anti-desmogleína e lesões mucocutâneas em pacientes com pênfigo vulgar ou foliáceo
}

\section{Correlation between anti-desmoglein and mucocutaneous lesions in patients with pemphigus vulgaris or foliaceus}

\section{Correlación entre anti-desmogleína y lesiones mucocutáneas en pacientes con pénfigo vulgar o foliáceo}

\section{Recebido: 03/05/2018 \\ Aprovado: 23/11/2018 Publicado: 29/01/2019}

Mara Ilka Holanda Medeiros Batista1

Marcilia Ribeiro Paulino ${ }^{2}$ Carlus Alberto Oliveira dos Santos ${ }^{3}$ Samantha Cardoso de Andrade ${ }^{4}$ Camila Andrade Lima Arcoverde 5 Luiz Alcino Monteiro Gueiros 6 Jair Carneiro Leão ${ }^{7}$ Alessandra Albuquerque Tavares Carvalho ${ }^{8}$

Essa é uma pesquisa não-probabilística e transversal. 0 objetivo deste estudo foi correlacionar a imunodetecção de anti-desmogleína 1 e 3 no soro de pacientes com pênfigo vulgar (PV) ou pênfigo foliáceo (PF) com a presença de lesões mucocutâneas. Pacientes foram selecionados por uma amostra de conveniência baseada na demanda espontânea nas unidades de Dermatologia e Medicina Oral da Universidade Federal do Pernambuco, Recife, Brasil, de fevereiro a novembro de 2012. Vinte e seis indivíduos (18 mulheres, 69,2\% e 8 homens, 30,8\%) foram avaliados, 20 diagnosticados com PV (73,1\%) e 6 com PF (26,9\%). 0 teste ELISA foi usado para determinar a presença de antidesmogleína 1 e 3 no soro. A presença de anti-desmogleína 1 foi associada a lesões na pele $(p=0,038)$ e a de antidesmogleína 3 a lesões mucosais $(p=0,041)$. Nesse estudo, o ELISA se mostrou altamente sensível ao DSG1 e ao DSG3, de acordo com o fenótipo da doença.

Descritores: Pênfigo; Ensaio de Imunoadsorção Enzimática; Dermatopatias Vesiculobolhosas; Doenças Autoimunes.

This research was a non-probability cross-sectional. The aim of the present study was to correlate the immunodetection of anti-desmoglein 1 and 3 in the serum of pemphigus vulgaris (PV) or pemphigus foliaceus (PF) patients with the presence of mucocutaneous lesions. Patients were selected through convenience sampling based on spontaneous demand at the Dermatology and Oral Medicine units of the Universidade Federal de Pernambuco, Recife, Brazil at February until November of 2012. Twenty-six individuals (18 women, 69.2\% and 8 men, 30.8\%) were evaluated, 20 diagnosed with PV (73.1\%) and 6 with PF (26.9\%). ELISA test was used to determine the presence of anti-desmoglein 1 and 3 in the serum. Anti-desmoglein 1 positivity was associated to skin lesions $(p=0.038)$ and antidesmoglein 3 to mucosal lesions $(p=0.041)$. On this study, ELISA was shown to be highly sensitive for DSG1 and DSG3 in accordance with the phenotype of the disease.

Descritores: Pênfigo; Ensaio de imunoadsorção enzimática; Dermatopatias vesicolobolhosas; Doenças autoimunes.

Esta es una investigación no-probabilística y transversal. El objetivo de este estudio fue correlacionar la inmunodetección de anti-desmogleína 1 y 3 en el suero de pacientes con pénfigo vulgar (PV) o pénfigo foliáceo (PF) con la presencia de lesiones mucocutáneas. Pacientes fueron seleccionados por una muestra de conveniencia basada en la demanda espontanea en las unidades de Dermatología y Medicina Oral de la Universidad Federal de Pernambuco, Recife, Brasil, de febrero a noviembre de 2012. Veintiséis individuos (18 mujeres, 69,2\% y 8 hombres, 30,8\%) fueron evaluados, 20 diagnosticados con PV $(73,1 \%)$ y 6 con PF $(26,9 \%)$. El test ELISA fue usado para determinar la presencia de anti-desmogleína 1 y 3 en el suero. La presencia de anti-desmogleína 1 fue asociada a lesiones en la piel $(p=0,038)$ y de anti-desmogleína 3 a lesiones mucosas $(p=0,041)$. En este estudio, el test ELISA se mostró altamente sensible al DSG1 y al DSG3, de acuerdo con el fenotipo de la enfermedad.

Descriptores: Pénfigo; Ensayo de inmunoadsorción enzimática; Enfermedades cutáneas Vesiculoampollosas; Enfermedades autoinmunes

1. Cirurgiã-Dentista. Especialista em Odontologia. Especialista em Ortodontia. Especialista em Estética e Cosmética em Odontologia. Mestre em Perícias Forenses. Doutoranda em Odontologia pela Universidade Federal de Pernambuco (UFPE). Professora assistente do curso de Odontologia do Centro Universitário de João Pessoa (UNIPÊ), João Pessoa, PB, Brasil. ORCID: 0000-0002-7314-0595 E-mail: marailka@hotmail.com

2. Cirurgião Dentista. Mestre em Odontologia. Doutoranda em Odontologia pela UFPE. Professora Substituta de Prótese na Universidade Leão Sampaio (Unileão), Juazeiro do Norte, CE, Brasil. ORCID: 0000-0002-3924-4251 E-mail: marcilia.paulino@yahoo.com.br

3. Cirurgião Dentista. Mestrando em Odontologia pela Universidade Estadual da Paraíba (UEPB), João Pessoa, PB, Brasil. ORCID: 0000-0002-5988-1186 E-mail: carlusalberto94@gmail.com

4. Cirurgiã-Dentista. Mestre em Odontologia. Doutoranda em Odontologia pela UFPE. Professora Substituta de Periodontia pela UFPE, Recife, PE, Brasil. ORCID: 0000-0003-0859-3499 E-mail: samanthadeandrade@hotmail.com

5. Cirurgiã-Dentista. Especialista em Implantodontia. Mestre em Odontologia. Recife, PE, Brasil. ORCID: 0000-0003-0522-919X E-mail: camila.arcoverde@gmail.com

6. Cirurgião Dentista. Especialista em Estomatologia. Mestre em Odontologia. Doutor em Estomatopatologia. Pós-Doutor em Odontologia. Professor Adjunto III da Disciplina de Estomatologia do Departamento de Clínica e Odontologia Preventiva e do Programa de Pós Graduação em Odontologia da UFPE, Recife, PE, Brasil. ORCID: 0000-0003-4979-4318 Email: lagueiro@gmail.com

7. Cirurgião Dentista. Mestre, Doutor e Pós-Doutor em Odontologia. Professor Titular do Curso de Odontologia da UFPE, Recife, PE, Brasil. ORCID: 0000-0002-1054-0305 E-mail: jleao@ufpe.com

8. Cirurgiã-Dentista. Doutora em Odontologia. Professora e Coordenadora do Programa de Pós-Graduação em Odontologia da UFPE, Recife, PE, Brasil. ORCID: 0000-0003-0925-7809 E-mail: alessandra.atcarvalho@gmail.com 


\section{INTRODUÇÃO}

$\mathbf{P}$ ênfigo se refere a um grupo de doenças autoimunes vesicobolhosas, incluindo pênfigo vulgar (PV), pênfigo foliáceo (PF), pênfigo vegetante, pênfigo paraneoplásico, pênfigo por imunoglobina $\mathrm{A}$, pênfigo eritematoso e pênfigo por drogas. Esse grupo de enfermidades é caracterizado pela perda da adesão intraepitelial (acantólise), pela formação de bolhas e úlceras afetando as membranas mucosas e/ou pele, e a presença de anticorpos direcionados às desmogleínas (DSG) ${ }^{1-3}$.

Os autoanticorpos produzidos no pênfigo são direcionadas às DSG encontradas na superfície dos queratinócitos, causando a separação de células epiteliais e levando a bolhas intraepiteliais ${ }^{2}$. As DSG pertencem a uma família de caderinas que agem como moléculas de adesão intercelular, unindo queratinócitos epidérmicos ${ }^{2,4,5}$.

A DSG1 é mais comumente encontrada na camada superficial enquanto a DSG3 é mais abundante em camadas basais e suprabasais. Além disso, a DSG3 se expressa de maneira significativa no epitélio oral ${ }^{6}$. Essa distribuição explica porque a PV afeta a pele e as membranas mucosas, enquanto a PF normalmente é vista na forma de lesões cutâneas $^{1-3,7}$. Clinicamente, a boca pode ser o primeiro e único local de acometimento do $\mathrm{PV}$, levando ao diagnóstico tardio e ao tratamento inadequado ${ }^{6}$.

$\mathrm{O}$ PV e o PF são os subtipos mais comuns de pênfigo, com uma incidência estimada de 0,1 a 0,5 casos a cada 100.000 habitantes para o primeiro, e cerca de 0,5 casos a cada 100.000 habitantes para o segundo ${ }^{8,9}$. Em regiões endêmicas, a prevalência de PF pode atingir $3,4 \%{ }^{10}$. Além disso, eles apresentam características clínicas e imunopatológicas distintas ${ }^{1}$. Ainda assim, a coexistência de características de PF e PV no mesmo paciente, ou mesmo a transformação de um tipo em outro, já foram bem documentadas ${ }^{11-14}$. Essa possibilidade existe pois pacientes com PV produzem imunoglobulina G (IgG) anti-DSG3 e/ou antiDSG1, enquanto pacientes com PF produzem apenas IgG anti-DSG113,4,7.
Nesse contexto clínico, a detecção dos anticorpos que circulam parece ser mais relevante para um diagnóstico adequado do pênfigo ${ }^{1,11,15}$. Por muitos anos, a imunofluorescência indireta foi a técnica mais usada para esse objetivo. Contudo, ela não diferencia entre os subtipos da doença, o que pode ser feito por meio de immunoblotting e imunoprecipitação. Infelizmente, esses métodos levam um longo tempo, são exclusivamente qualitativos, e pouco práticos para uso em um grande número de $\operatorname{casos}^{2,9,16}$.

A produção dos antígenos recombinantes de DSG1 e DSG3 nos anos 1990 levou ao desenvolvimento de um ensaio de imunoadsorção enzimática (ELISA, na sigla em inglês), capaz de identificar os anticorpos do pênfigo ${ }^{17-20}$. Esse teste demonstrou uma elevada sensibilidade e especificidade no diagnóstico de PV quando comparado com outros testes sorológicos, sendo capaz de detectar e quantificar os anticorpos, o que parece se relacionar à severidade da doença ${ }^{9,15}$.

Detectar a presença dos anticorpos circunlantes é uma realidade no diagnóstico de pênfigo, resultando na importância de se conduzir estudos a respeito. Portanto, o objetivo deste estudo foi correlacionar a imunodetecção de anti-desmogleína 1 e 3 no soro de pacientes com PV ou PF com a presença de lesões mucocutâneas.

\section{MÉTODO}

Essa é uma pesquisa não-probabilística e transversal. Pacientes foram selecionados por uma amostra de conveniência baseada na demanda espontânea nas unidades de Dermatologia e Medicina Oral da Universidade Federal do Pernambuco (Recife, Brasil), de fevereiro a novembro de 2012.

0 estudo foi realizado levando em consideração os princípios éticos estipulados pelo Conselho Nacional de Saúde brasileiro e foi aprovado pelo conselho da instituição sob número 291/08. Todos os participantes assinaram o termo de consentimento livre e esclarecido. 
Todos os pacientes haviam sido diagnosticados previamente e tratados na Unidade de Dermatologia. Os diagnósticos foram baseados na apresentação clínica e nos aspectos microscópicos. Pacientes foram entrevistados e exames físicos foram realizados na Unidade de Medicina Oral, UFPE. Amostras de sangue foram obtidas, colocadas em tubos de $9 \mathrm{~mL}$ e deixadas em descanso por $30 \mathrm{~min}$. Depois desse período, os tubos foram centrifugados por dez minutos a $3250 \mathrm{rpm}$, e o soro foi transferido para microtubos estéreis e etiquetados, que foram armazenados a $-20^{\circ} \mathrm{C}$ até a análise.

A detecção de DSG1 e DSG3 foi realizada usando o Sistema de Exames ELISA MBL Mesacup DSG-1 e DSG-3® ${ }^{\odot}$ (Medical and and Biological Laboratories - MBL ${ }^{\circledR}$, Nagoya, Japão), de acordo com as instruções do fabricante. Resumidamente, $100 \mu$ de uma amostra de soro sanguíneo diluída (1:101) de cada paciente foi depositada em duplicata na microlâmina. Controles positivo e negativo para cada desmogleína foram usados e as microlâminas foram incubadas por 60 minutos a temperaturas de $23 \pm 0,3^{\circ} \mathrm{C}$, lavadas quatro vezes, e então o conjugado de peroxidase com IgG foi adicionado. Depois de 60 min, a lâmina foi lavada e o substrato de peroxidase foi adicionado (solução de substrato de TMB) por 30 minutos, e então $100 \mu \mathrm{l}$ da solução de parada. Uma leitura imediata foi realizada utilizando um espectrofotômetro (TP-Reader Plus, Thermoplate) a $450 \mathrm{~nm}$. Resultados são dados em absorbância (Abs), e a concentração $(\mathrm{U} / \mathrm{mL})$ foi determinada de acordo com a fórmula do fabricante.

Esse resultado foi interpretado utilizando a tabela indicativa de pontos de corte recomendada pelo fabricante, que quantifica os resultados como positivos, negativos ou indeterminados, como na Tabela 1.

Tabela 1. Tabela de pontos de corte recomendados pelo fabricante

\begin{tabular}{c|c}
\hline DSG $1<14 \mathrm{U} / \mathrm{mL}$ & Negativo \\
DSG3 $<9 \mathrm{U} / \mathrm{mL}$ & Indeterminado \\
\hline $14<\mathrm{DSG} 1<20 \mathrm{U} / \mathrm{mL}$ & Positivo \\
\hline DSG1 e DSG3 $>20 \mathrm{U} / \mathrm{mL}$ & \\
\hline
\end{tabular}

Abreviações: DSG1, Desmogleína tipo 1; DSG3, Desmogleína tipo 3.

Como análise univariada foram obtidas as distribuições absolutas e relativas, e a análise bivariada foi feita através do teste de qui-quadrado. Cálculos estatísticos foram feitos usando o Statistical Package for the Social Sciences (SPSS - version 17.0). A margem de erro usada nas decisões dos testes estatísticos foi de 5,0\%.

\section{RESULTADOS}

A amostra incluiu 26 indivíduos, com idade média de 43,2 anos, variando de 14 a 74 anos. Dezoito participantes eram do sexo feminino (69.24\%) e oito do masculino $(30,76 \%)$. Vinte tinham PV $(73,1 \%)$ e seis tinham PF $(26,9 \%)$. Todos haviam nascido no estado de Pernambuco, Brasil. Os diagnósticos foram realizados por meio de exames clínicos e histopatológicos. No momento do exame clínico, 18 (69,23\%) dos
26 pacientes tinham lesões ativas e 18 $(69,23 \%)$ estavam sendo tratados com corticosteroides sistêmicos.

Pode-se observar que entre os 26 pacientes diagnosticados com pênfigo, vinte $(73,1 \%)$ tinham PV e seis $(26,9 \%)$ tinham PF. Quinze pacientes $(57,7 \%)$ tinham um histórico de lesões mucocutâneas, 7 (26,9\%) tinham apenas lesões na pele, e em 4 casos $(15,4 \%)$ havia apenas lesões mucosais. A tabela 2 mostra a relação entre a presença de anti-DSG1 e os subtipos da doença. Resumidamente, dois pacientes (10\%) com PV apresentaram anti-DSG1, sete (35\%) apresentaram anti-DSG3, e sete (35\%) apresentaram ambas. Entre os seis pacientes com $\mathrm{PF}$, cinco $(83,3 \%)$ eram positivos para anti-DSG1 e um $(16,7 \%)$ teve um resultado negativo (Tabela 2). 
Tabela 2. Correlação entre a positividade de Anti-DSG1 e Anti-DSG3 e a doença. Recife, Brasil, 2012.

\begin{tabular}{c|c|c|c|c}
\hline Doença & \multicolumn{4}{|c}{ DSG } \\
\hline & $\begin{array}{c}\text { DSG-1 } \\
\text { Positivo }\end{array}$ & $\begin{array}{c}\text { DSG-3 } \\
\text { Positivo }\end{array}$ & $\begin{array}{c}\text { DSG-1 } \\
\text { Negativo }\end{array}$ & $\begin{array}{c}\text { DSG-3 } \\
\text { Negativo }\end{array}$ \\
\hline Pênfigo vulgar & 7 & 11 & 6 & 4 \\
\hline Pênfigo foliáceo & 7 & 3 & 2 & 5 \\
\hline Total & 14 & 14 & 8 & 9 \\
\hline
\end{tabular}

Abreviações: DSG1, Desmogleína tipo 1; DSG3, Desmogleína tipo 3.

A presença de anticorpos Anti-DSG1 foi associada a lesões mucosais e na pele $(\mathrm{p}=0,038$ e $\mathrm{p}=0,009$, respectivamente). Entre os 19 pacientes com um histórico de lesões na pele, oito $(30,8 \%)$ tiveram resultados positivos para a anti-DSG1 (Table 3). De 22 pacientes com lesões mucosais, 14 tiveram resultados positivos para anti-DSG1 (Tabela 4). Não foi encontrada relação estatisticamente significativa entre a atividade do anti-DSG3 e lesões na pele $(p=0,850)$, enquanto uma correlação estatisticamente significativa foi encontrada entre o anti-DSG3 positivo e lesões mucosais $(\mathrm{p}=0,041)$ (Tabelas 3 e 4). Entre os 22 pacientes com um histórico de lesões mucosais, em 13 o resultado dos exames foi positivo para a presença de anti-DSG3 (Tabela 4).

Tabela 3. Correlação entre Anti-DSG1/DSG3 positivas e a presença de lesões na pele. Recife, Brasil, 2012.

\begin{tabular}{l|c|c|c|c|c|c}
\hline \multirow{2}{*}{$\begin{array}{l}\text { Lesões na } \\
\text { pele }\end{array}$} & \multicolumn{7}{|c|}{ DSG } & \multicolumn{1}{c}{} \\
\cline { 2 - 7 } & $\begin{array}{c}\text { Anti- } \\
\text { DSG1 }\end{array}$ & $\begin{array}{c}\text { Anti- } \\
\text { DSG3 }\end{array}$ & $\begin{array}{c}\text { Ambas } \\
\text { positivas }\end{array}$ & $\begin{array}{c}\text { Ambas } \\
\text { negativas }\end{array}$ & Total & $P$ \\
\hline Presente & 2 & 6 & 6 & 5 & 19 & 0,038 \\
\hline Ausente & 5 & 1 & 1 & 0 & 7 & \\
\hline Total & 7 & 7 & 7 & 5 & 26 & \\
\hline
\end{tabular}

Abreviações: DSG1, Desmogleína tipo 1; DSG3, Desmogleína tipo 3.

Tabela 4. Correlação entre Anti-DSG1/DSG3 positivas e lesões da mucosa. Recife, Brasil, 2012.

\begin{tabular}{l|c|c|c|c|c|c}
\hline \multirow{2}{*}{$\begin{array}{l}\text { Lesões da } \\
\text { mucosa }\end{array}$} & \multicolumn{7}{c}{ DSG } & Negativo & Total & $P$ \\
\cline { 2 - 7 } & $\begin{array}{c}\text { Anti- } \\
\text { DSG1 }\end{array}$ & $\begin{array}{c}\text { Anti- } \\
\text { DSG3 }\end{array}$ & Ambas & 6 & 23 & 0,009 \\
\hline Presente & 4 & 7 & 6 & 2 & 3 & \\
\hline Ausente & 0 & 1 & 0 & 8 & 26 & \\
\hline Total & 4 & 8 & 6 &
\end{tabular}

\section{DISCUSSÃO}

PV e PF são doenças bolhosas da pele e/ou membrana mucosa, caracterizadas pela circulação de anticorpos IgG contra DSG1 e/ou DSG317. A PF é endêmica em certas áreas brasileiras e outros países subtropicais 16,21,22. A PV é o subtipo mais comum de pênfigo, no qual lesões se desenvolvem na membrana mucosa e/ou na pele ${ }^{14}$.

Nesse estudo, $73,1 \%$ dos pacientes sofriam de PV. No presente estudo, a maior parte da amostra $(69,23 \%)$ era composta por pacientes do sexo feminino, a maioria entre 24 e 62 anos de idade. Esses resultados parecem estar de acordo com outros estudos, que em geral relatam que o pênfigo é mais comum entre mulheres de 15 a 34 anos $^{8}$.

DSG3 e DSG1 são glicoproteínas 130 kDa e 160 kDa membras da superfamília de caderinas desmossomais. Essas proteínas são organizadas e concentradas nos desmossomos, sendo responsáveis por manter a integridade do epitélio estratificado $^{7,19}$. Elas também são os antiantígenos mais comuns para PV (DSG3) e PF (DSG1)1,2,5,23. A DSG3 é um determinante de lesões da mucosa e a DSG1 é um determinante de lesões na pele.

Pacientes com PV e lesões mucocutâneas podem exibir tanto anticorpos 
anti-DSG1 quanto anti-DSG34,9,21,23,24, e mais de $50 \%$ dos pacientes apresentam ambos DSG1 e DSG3. Estudos sugerem que esse é um importante fator ao se determinar o fenótipo da doença, uma vez que pacientes sofrendo predominantemente de lesões na pele tem níveis de anticorpos DSG1 mais altos quando comparados àqueles com lesões predominantemente mucosais ${ }^{4,7,24}$.

Neste estudo, o ELISA para DSG1 e DSG3 provou ser uma ferramenta de alta sensibilidade e especificidade para o diagnóstico de pênfigo. Essa descoberta corrobora numerosos outros estudos, que relatam que o ELISA para DSG1 e DSG3 é altamente sensível e também pode ser usado para avaliar a severidade e a atividade da doença7,18,21. O ELISA tem muitas outras vantagens sobre o método da imunofluorescência indireta, pois não requer um observador qualificado e é uma maneira simples de diferenciar entre $\mathrm{PV}$ e $\mathrm{PF}^{21}$.

Nesse estudo, todos os pacientes com PF apresentaram lesões de pele e resultados positivos para DSG1, enquanto a maioria dos pacientes com PV (75\%) apresentaram lesões mucocutâneas e testaram positivo para ambos DSG1 e DSG3. O perfil de desmogleína parece ser consistente com o fenótipo da doença. Ainda que o fenótipo clínico de uma doença em geral seja relacionado ao tipo de anticorpo, há alguns casos em que o fenótipo e o anticorpo são diferentes. Tais discrepâncias podem se dever a variações genéticas ou à presença de outros antígenos envolvidos na patogênese do pênfigo ${ }^{24}$. Curiosamente, 3 pacientes com PF foram positivos para ambos DSG1 e DSG3. Isso pode se associar a uma mudança no fenótipo de PF para PV, como mencionado previamente ${ }^{11-14}$.

0 tratamento de pênfigo envolve uma alta dose de corticoesteroides sistêmicos, especialmente prednisona, que podem ser combinados com imunosupressores, tais como azatioprina, ciclofosfamida, metotrexato, ciclosporina, e, mais recentemente, mofetil micofenolato. Drogas anti-inflamatórias, tais como dapsona, cloroquina, e combinações de nicotinamida e tetraciclina também são usadas ${ }^{2,23}$.
Há alguns relatos de caso do uso de rituximabe em pacientes com pênfigo, especialmente em pênfigo vulgar resistente a esteroides e imunossupressores, com resultados favoráveis ${ }^{12}$. Essas são terapias adjuvantes, cujo objetivo é reduzir os efeitos colaterais frequentemente devastadores do tratamento com corticoesteroides ${ }^{2,10}$. 0 tratamento com prednisona frequentemente produz ótimos resultados, mas existem formas resistentes que requerem terapias alternativas.

Tratamentos alternativos têm sido utilizados com resultados favoráveis em casos de pênfigo refratário a corticoesteroides, tais como a imunoglobulina intravenosa ${ }^{11,12}$. No momento da realização do presente estudo, $73,1 \%$ dos pacientes estão passando por tratamentos sistêmicos com esteróides e controle adequado da apresentação da doença. Aqueles sem tratamento sistêmico estão clinicamente estáveis sem tratamento ou utilizando esteróides tópicos.

Esse estudo tem limitações devido à amostra por conveniência e a metodologia utilizada (transversal). Também vale notar que os resultados encontrados são locais, o que levanta novas questões para o estudo que permitem a extrapolação de resultados para a população em geral.

\section{CONCLUSÃO}

Em resumo, o ELISA se mostrou altamente sensível ao DSG1 e ao DSG3, de acordo com o fenótipo da doença. Além disso, correlações foram encontradas entre a presença de antiDSG1 e lesões na pele, assim como entre antiDSG3 e lesões na mucosa. Do mesmo modo, ambos anti-DSG1 e anti-DSG3 foram encontrados em pacientes com lesões mucocutâneas.

\section{REFERÊNCIAS}

1. De D, Khullar G, Handa S, Joshi N, Saikia B, Minz RW. Correlation between salivary and serum anti-desmoglein 1 and 3 antibody titres using ELISA and between antidesmoglein levels and disease severity in pemphigus vulgaris. Clin Exp Dermatol. 2017; 42(6):648-50. 
2. Feller L. Immunopathogenic oral diseases: an overview focusing on pemphigus vulgaris and mucous membrane pemphigoid. Oral Health Prev Dent. 2017; 15(2):177-82.

3. Ruocco V, Ruocco E, Lo Schiavo A, Brunetti G, Guerrera LP, Wolf R. Pemphigus: etiology, pathogenesis, and inducing or triggering factors: facts and controversies. Clin Dermatol. 2013; 31(4):374-81.

4. Santosh ABR, Addam VRR. Oral mucosal lesion in pa/ents with pemphigus and pemphigoid skin diseases: across sec/onal study from southern India. Dentistry 3000. 2017; 5(1):1-7.

5. Perks AC. A case of concomitant pemphigus foliaceus and oral pemphigus vulgaris. Head Neck Pathol. 2018; 1(1):1-6.

6. Tamgadge $S$, Tamgadge $A$, Bhatt $D M$, Bhalerao S, Pereira T. Pemphigus vulgaris. Contemp Clin Dent. 2011; 2(2):134-7.

7. Neumann-Jensen B, Worsaae N, Dabelsteen E, Ullman S. Pemphigus vulgaris and pemphigus foliaceus coexisting with lichen planus. $\mathrm{Br} J$ Dermatol. 1980; 102(5):585-90.

8. Sharma M. Oral pemphigus vulgaris. J Kathmandu Med Coll. 2015; 4(3):100-3.

9. Patsatsi A, Kyriakou A, Giannakou A, Pavlitous-Tsiontsi A, Lambropoulos A, Dimitrios Sotiriadis D. Clinical significance of anti-desmoglein -1 and -3 circulating autoantibodies in pemphigus patients measured by Area Index and Intensity Score. Acta Derm Venereol. 2014; 94(2):203-6.

10. Reeves GMB, Lloyd M, Rajlawat BP, Barker GL, Field EA, Kaye SB. Ocular and oral grading of mucous membrane pemphigoid. Graefes Arch Clin Exp Ophthalmol. 2012; 250(4):611-8.

11. Ni Riordain R, Shirlaw P, Alajbeg I. World Workshop on Oral Medicine VI: patient reported outcome measures and oral mucosal disease: current status and future direction. Oral Surg Oral Med Oral Pathol Oral Radiol. 2015; 120(2):161-71.e20.

12. Di Zenzo G, Carrozzo M, Chan LS. Urban legend series: mucous membrane pemphigoid. Oral Dis. 2014; 20(1):35-54.

13. Izumi T, Seishima $M$, Satoh $S$, Ito $A$, Kamija H, Kitajima Y. Pemphigus with features of both vulgaris and foliaceus variants associated with antibodies to 160 and $130 \mathrm{kDa}$ antigens. Br J Dermatol. 1998; 139(4):688-92.

14. Cunha PR, Bystryn JC, Medeiros EPL, Oliveir,a JR. Sensitivity of indirect immunofluorescence and ELISA in detecting intercellular antibodies in endemic pemphigus foliaceus. Int J Dermatol. 2006; 45(8):914-8.

15. Oiso N, Yamashita C, Yoshioka K, Amagai M, Komai A, Nagata A. IgG/IgA pemphigus with IgG and IgA antidesmoglein 1 antibodies detected by enzyme-linked immunosorbent assay. Br J Dermatol. 2002; 147(5):1012-7.

16. Ishii $K$, Amagai $M$, Hall RP, Hashimoto $T$, Takayanagi A, Shimizu A. Characterization of autoantibodies in pemphigus using antigenspecific enzyme-linked immunosorbent assays with baculovirus expressed recombinant desmogleins. J Immunol. 1997; 159(4):2010-7.

17. Mortazavi H, Khatami A, Seyedin Z, Farahani IV, Daneshpazhoohi M. Salivary desmogleína enzyme-linked immunosorbent assay for diagnosis of pemphigus vulgaris: a noninvasive alternative test to serum assessment. Biomed Res. Int. [Internet]. 2015 [citado em 14 out 2017]; ID 698310:1-7. Disponível em: https://www.hindawi.com/journals/bmri/2 015/698310/

18. Sami N, Bhol C, Ahmed AR. Diagnostic features of pemphigus vulgaris in patients with pemphigus foliaceus: detection of both autoantibodies, long-term follow-up and treatment responses. Clin Exp Immunol. 2001; 125(3):492-8.

19. Shamim T, Varghese VI, Shameena PM, Sudha S. Pemphigus vulgaris in oral cavity: clinical analysis of 71 cases. Med Oral Patol Oral Cir Bucal. 2008; 13(10):E622-6.

20. Amagai M. Desmoglein as a target in autoimmunity and infection. J Am Acad Dermatol. 2006; 48(2):244-52.

21. Kouskoukis CE, Ackerman AB. Vacuoles in the upper part of the epidermis as a clue to eventuation of superficial pemphigus and bullous impetigo. Am J Dermatopathol. 1984; 6(2):183-6.

22. Ito T, Moriuchi R, Kikuchi K, Shimizu S. Rapid transition from pemphigus vulgaris to 
pemphigus foliaceus. J Eur Acad Dermatol Venereol. 2016; 30(3):455-7.

23. McMillan R, Taylor J, Shephard M, Ahmed R, Carrozzo M, Setterfield J, et al. World Workshop on Oral Medicine VI: a systematic review of the treatment of mucocutaneous pemphigus vulgaris. Oral Surg Oral Med Oral Pathol Oral Radiol. 2015; 120(2):132-42.

24. Teixeira TA, Fiori FCBC, Silvestre MC, Borges CB, Maciel VG, Costa MB. Refractory endemic pemphigus foliaceous in adolescence successfully treated with intravenous immunoglobulin. An Bras Dermatol. 2011; 86(4Suppl 1):S133-6.

CONTRIBUIÇÕES
Mara Ilka Holanda Medeiros Batista e
Marcilia Ribeiro Paulino atuaram na
concepção, delineamento e redação. Carlus
Alberto Oliveira dos Santos contribuiu na
revisão e redação. Samantha Camargo de
Andrade e Camila Andrade de Lima
participaram na elaboração do projeto e
coleta de dados. Luiz Alcino Gueiros e Jair
Carneiro Leão contribuíram na análise dos
dados. Alessandra Albuquerque Tavares
Carvalho atuou na orientação e revisão
crítica.

Mara Ilka Holanda Medeiros Batista e Marcilia Ribeiro Paulino atuaram na concepção, delineamento e redação. Carlus Aiberto Oliveira dos Santos contribuiu na revisão e redação. Samantha Camargo de Andrade e Camila Andrade de Lima participaram na elaboração do projeto e coleta de dados. Luiz Alcino Gueiros e Jair Carneiro Leão contribuíram na análise dos dados. Alessandra Albuquerque Tavares crítica.

\footnotetext{
Como citar este artigo (Vancouver)

Batista MIHM, Paulino MR, Santos CAO, Andrade SC, Arcoverde CAL, Gueiros LA, et al. Correlação entre anti-desmogleína e lesões mucocutâneas em pacientes com pênfigo vulgar ou foliáceo REFACS [Internet]. 2019 [citado em inserir dia, mês e ano de acesso]; 7(1):14-20. Disponível em: inserir link de acesso. DOI: inserir link do DOI.

Como citar este artigo (ABNT)

BATISTA, M. I. H. M. et al. Correlação entre anti-desmogleína e lesões mucocutâneas em pacientes com pênfigo vulgar ou foliáceo REFACS, Uberaba, MG, v. 7, n. 1, p. 14-20, 2019. Disponível em: <inserir link de acesso>. Acesso em: inserir dia, mês e ano de acesso. DOI: inserir link do DOI.

Como citar este artigo (APA)

Batista, M.I.H.M., Paulino, M.R., Santos, C.A.O., Andrade, S.C., Arcoverde, C.A.L, Gueiros, L.A., ... Carvalho, A.A.T. (2019). Correlação entre anti-desmogleína e lesões mucocutâneas em pacientes com pênfigo vulgar ou foliáceo REFACS, 7(1), 14-20. Recuperado em: inserir dia, mês e ano de acesso de inserir link de acesso. DOI: inserir link do DOI.
} 